\title{
Essential Quality Analysis Criteria in Forensic Genetics Identification: A Position Statement of Italian Society of Human Genetics
}

Giardina Emiliano*1,2, Piluso Giulio ${ }^{3}$, Del Vecchio Blanco Francesca ${ }^{3}$, Nutini Anna Lucia ${ }^{4}$, Ragazzo Michele ${ }^{1}$, Manzo Laura' ${ }^{1}$, Binni Francesco ${ }^{5}$, D’Ambrosio Anna ${ }^{5}$, Di Natale Manuela ${ }^{6}$, Pietrangeli Ilenia ${ }^{7}$, Piumelli Nunzia ${ }^{7}$, Zampatti Stefania ${ }^{2,8}$, Torrente Isabella ${ }^{9}$, Mari Francesca ${ }^{10}$, Bruttini Mirella ${ }^{11,12}$, Amitrano Sara ${ }^{13}$, Raso Barbara $^{14}$, Salvaderi Luca ${ }^{15}$, Novelli Giuseppe ${ }^{1}$ and Grammatico Paola ${ }^{5}$

${ }^{1}$ Department of Biomedicine and Prevention, School of Medicine, University of Rome "Tor Vergata", Rome, Italy

${ }^{2}$ Laboratory of Genomic Medicine, Fondazione Santa Lucia IRCCS, Rome, Italy

${ }^{3}$ Department of Precision Medicine, University of Campania "Luigi Vanvitelli", Italy

${ }^{4}$ Diagnostic Genetics Unit, Azienda Ospedaliero-Universitaria “Careggi”, Florence, Italy

${ }^{5}$ Medical Genetics, Molecular Medicine Department, Sapienza University of Rome, San Camillo-Forlanini Hospital, Rome, Italy

${ }^{6}$ Department of Biology, University of Naples Federico II, Naples, Italy

${ }^{7}$ Central Laboratory of National DNA Database, Department of Penitentiary Administration, Ministry of Justice, Rome, Italy

${ }^{8}$ IRCCS Neuromed, Località Camerelle, Pozzilli, Italy

${ }_{9}^{9}$ Mendel Laboratory, Casa Sollievo della Sofferenza Hospital, IRCCS, San Giovanni Rotondo (FG), Italy

${ }^{10}$ Medical Genetics, Department of Medical Biotechnologies, University of Siena, Siena, Italy

${ }^{11}$ Medical Genetics, University of Siena, Policlinico Le Scotte, Siena, Italy

${ }^{12}$ Medical Genetics, Azienda University Hospital, Siena, Italy

${ }^{13}$ Medical Genetics, Azienda Ospedaliera Universitaria Senese, Siena, Italy

${ }^{14}$ Servizio di Genetica Medica, Clinica Sant'Anna, Rome, Italy

${ }^{15}$ Genoma Group, Forensic Unit, Milan, Italy

Received: 㯺: December 05, 2018; Published: 眥: December 14, 2018

*Corresponding author: Emiliano Giardina, University of Rome Tor Vergata, via Montpellier, 100133, Rome

Abstract

The entry into force of DPR n. 87 of $7^{\text {th }}$ April 2016 relating to the DNA Data Bank requires an implementation upgrade of the Forensic Genetics analysis to ensure the quality of results by the control of the pre-analytical and analytical processes. The decree provides the opportunity to define the operational guidance elements, shared by Italian laboratories in the Forensic Genetics field, aimed at ensuring the quality of data and at producing useful genetic profiles: for this reason, the laboratories who want to contribute to the Bank of DNA data need accreditation in accordance with ISO/ IEC 17025. This document represents a brief technical document of the Italian Society of Human Genetics in the aim of supporting laboratories seeking accreditation as well as the Italian National Accreditation Body appointed by the State to perform accreditation activity (ACCREDIA).

Keywords: Criteria of Analysis; Forensic Genetics; Accreditation Activity; ISO

\section{Introduction}

The entry into force of DPR n. 87 of 7th April 2016 relating to the DNA Data Bank requires an implementation upgrade of the Forensic Genetics analysis to ensure the quality of results by the control of the pre-analytical and analytical processes. The decree provides the opportunity to define the operational guidance elements, shared by Italian laboratories in the Forensic Genetics field, aimed at ensuring 
the quality of data and at producing useful genetic profiles: for this reason, the laboratories who want to contribute to the Bank of DNA data need accreditation in accordance with ISO/IEC 17025. In Italy few laboratories have been accredited so far. For this reason, the SIGU Working Group on Forensic Genetics perspective is to promote the accreditation path of Forensic Genetics laboratories. This document represents a brief technical document of the Italian Society of Human Genetics in the aim of supporting laboratories seeking accreditation as well as the Italian National Accreditation Body appointed by the State to perform accreditation activity (ACCREDIA).

\section{Acceptance and Management of Reference/Evidence Samples}

a) Criteria 1: All pre-analytical procedures (sample collection methods, inspection methods and evidence conservation, etc.), analytical procedures (methods of extraction, quantification, typing etc.) and post-analytical procedures (data interpretation) must be documented.

b) Criteria 2: All the files, worksheets, raw data, photographs, etc. related to a single procedure must be saved as directed by the judicial authorities. The DNA extracts must be available for the judicial authority, who are responsible for the authorization of their destruction, storage or return.

c) Criteria 3: It is necessary that the laboratory ensures and maintains the integrity of the chain of custody of any evidence. The procedure is to write a record of acceptance documenting the origin of the reference/evidence samples together with the identity of the person who delivers those items to the laboratory [1].

d) Criteria 4: It is necessary that the laboratory establishes eligibility criteria with respect to conservation, storage and transport of reference/evidence samples.

\section{Laboratory Structure and Staff}

a) Criteria 5: Only authorized persons are permitted access to the sample conservation and analysis locations.

b) Criteria 6: It is necessary to have a database containing the genetic profiles of people who have access to the lab for exclusion purposes. The database must be kept in accordance with any current regulations concerning the protection of privacy.

c) Criteria 7: The laboratory has to document the competence, training and experience of its staff. The Quality Manual has to include the minimum requirements for the staff (Degree in Biology / Biotechnology / Medicine; Postgraduate courses in Forensic Genetics; professional experience gained in forensic genetics laboratories).

\section{Analytical Workflow}

a) Criteria 8: All the results of the analyses used to determine the nature of the biological specimens (generic diagnoses) must be saved through image capture $[2,3]$. b) Criteria 9: It is preferable that the generic diagnosis includes negative and positive controls.

c) Criteria 10: It is preferable to have an automatic reader able to print a clear real-time stamped report, with accurate, test result quantification [4].

d) Criteria 11: In order to monitor the process and ensure the absence of contamination during the extraction of DNA, reagent blanks (extraction controls) must be used.

e) Criteria 12: It is preferable to use automated DNA extraction systems [2].

f) Criteria 13: It is preferable to use Real-Time technology for nucleic acid quantification from evidence samples. It is preferable to carry out DNA quantification for reference samples, except in the case of direct methods.

g) Criteria 14: The reagent blank (extraction control) must be quantified [5].

h) Criteria 15: It is necessary to use commercial amplification kits validated for forensic use [2].

i) Criteria 16: It is necessary to carry out at least one negative, and one positive control, for each amplification run [6].

j) Criteria 17: It is necessary to perform the amplification of the reagent blank (extraction control) [5].

k) Criteria 18: It is necessary to analyze samples and the allelic ladder in the same electrophoresis run.

1) Criteria 19: The reference and evidence samples must be analyzed in two separate and distinct test runs.

\section{Interpretation Step}

a) Criteria 20: It is necessary that the laboratory has defined, within its internal method, the acceptability criteria for negative and positive controls, reagent blank extraction and the allelic ladder [6-10].

b) Criteria 21: It is necessary that the laboratory has experimentally determined the analytical threshold (AT) $[6,11]$.

c) Criteria 22: It is necessary to use a probabilistic biostatistical method [12-14].

d) Criteria 23: It is necessary that the laboratory has experimentally defined the stochastic threshold (ST) required for the interpretation of single profiles [15].

e) Criteria 24: It is necessary that the laboratory has experimentally defined the value threshold LOQ (RFU), if the method of interpretation requires it [16].

f) Criteria 25: It is necessary that the all genetic profiles are compared through bio-statistical calculation to obtain the LR value (single and mixed profiles) $[17,18]$.

g) Criteria 26: In mixture profiles, it is necessary to calculate the LR value for each contributor separately [17-19]. 
h) Criteria 27: It is preferable that laboratories define the analytical characteristics of the profiles that require a confirmation by repetition of the amplification process $[15,20]$.

i) Criteria 28: It is necessary for the laboratory to participate in at least one annual proficiency testing (PT) [7].

\section{Technical Report}

a) Criteria 29: It is preferable to include electropherograms of all analyzed samples, including controls.

b) Criteria 30: It is preferable to include any available reader reports or images for the interpretation of the generic diagnostic results [4].

c) Criteria 31: It is necessary to provide the quantification results and it is preferable to include the associated instrument reports.

d) Criteria 32: It is necessary to include the results of biostatistical calculations and it is preferable to include the associated biostatistical software reports [19].

e) Criteria 33: It is necessary that the technical report declares the existence of, and conformity to, an accredited quality management system [21].

\section{Glossary}

Necessary: essential/mandatory criteria to guarantee the suitability of a process or a test.

Preferable: recommended criteria to ensure the best quality of a process or a test.

Proficiency Testing: Proficiency testing determines the performance of individual laboratories for specific tests or measurements and is used to monitor laboratories' continuing performance.

Biological Samples: samples with known biological origins (e.g. buccal swab, sampling of peripheral blood).

Evidence Samples: items of any kind, presumably related to a criminal event and to be subjected to forensic genetic analysis.

LR: The Likelihood Ratio: the relationship between two mutually exclusive and exhaustive hypotheses. The numerator ( $\mathrm{Hp}$ ) represents the hypothesis to be proven (e.g. The analyzed subject is the one who has contributed to the trace) and the denominator (Hd) represents the opposite scenario (e.g. A person unknown has contributed to the trace). The numerator hypothesis is more likely if the LR value is $>1$, while the denominator hypothesis is more likely if the $L R$ value is $<1$; if, instead, the LR value is 1 , the analysis is to be considered inconclusive [15].

Probabilistic Method: it is the method that considers the probability of occurrence of stochastic effects (drop-in and dropout). In case of non-identity between genotypes, this approach provides the hypothesis $\mathrm{Hp}$, a value not necessarily equal to 0 , as expected from the binary method, but a value between 0 and 1 [8].
Analytical Threshold: threshold value expressed in Relative Fluorescence Units (RFU), below which the highlighted signals are considered "background noise", above which the highlighted signals are considered in the interpretation.

Stochastic Threshold: threshold value expressed in Relative Fluorescence Units (RFU), above which you can exclude the stochastic phenomenon of "drop-out" associated with a homozygous allele.

LOQ: the threshold value measured in terms of Relative Fluorescence (RFU Unit), above which it is possible to ensure a direct correlation between the amount of DNA introduced into the PCR and intensity of the electrophoretic signals, in terms of RFU.

Units of Relative Fluorescence (RFU): relative measure of an allele in an electropherogram; it is directly proportional to the amount of amplicon present in the sample. The scale of the RFU is shown on the $y$-axis of an electropherogram.

Generic Diagnosis: analysis useful in evaluating the biological nature of the trace.

Raw Data: it is the data resulting from electrophoresis containing all the running parameters used in electrophoretic analysis.

Negative Control: sample devoid of biological material, but containing the reagents employed in the various test phases; it is used for the contamination monitoring.

Positive Control: biological sample of known genotypic profile; It is used for the confirmation of the proper functioning of analytical reagents.

Allelic Ladder: sample, containing the most common STR alleles present in the population. It is used to correlate the size (expressed in base pairs) of the amplification product with a unique reference system identical for all laboratories.

\section{Acknowledgment}

The authors would like to thank the other members of SIGU Working Group on Forensic Genetics for their contribution in debating this issue: Barone Chiara, Bianca Sebastiano, Caliendo Irene, Carta Piera, Civolani Alessandro, Di Stefano Cristina, Forzano Francesca, Fronduti Alessio, Iozzi Sara, Longo Ilaria, Maiello Angela, Mesoraca Alvaro, Migliosi Marina, Mucciolo Mafalda, Resta Nicoletta, Sepe Carlo, Steindl Katharina, Torricelli Francesca, Zanchetti Sara.

\section{References}

1. Lee HC, Ladd C (2001) Preservation and collection of biological evidence. Croatian Medical Journal 42(3): 225-228.

2. Butler JM (2011) Advanced topics in forensic DNA typing: Methodology. ( $1^{\text {st }}$ Edn.) Elsevier Academic Press.

3. US (1999) Department of Justice. Bureau of Alcohol, Tobacco and Firearms, Crime Scene and Evidence Collection Handbook.

4. Sinelnikov A, Kalinina A, Old JB, Boonlayangoor PW, Reich KA (2013) Evaluation of Rapid Stain Identification (RSID ${ }^{\text {TM }}$ ) Reader System for Analysis and Documentation of RSID ${ }^{\text {TM }}$ Tests. Appl Sci 3: 624-635. 
5. (2010) ENFSI DNA Working Group, Contamination prevention guidelines.

6. (2010) Scientific Working Group on DNA Analysis Methods (SWGDAM), Interpretation Guidelines for Autosomal STR Typing by Forensic DNA Testing Laboratories.

7. ISO/IEC 17025:2005 General requirements for the competence of testing and calibration laboratories.

8. ILAC G19:08/2014 Modules in a Forensic Science Process.

9. (2015) ENFSI DNA Working Group, Guideline for Evaluative Reporting in Forensic Science.

10. EURACHEM Guide (2014) The Fitness for Purpose of Analytical Methods. A Laboratory Guide to Method Validation and Related Topics.

11. Bregu J, Conklin D, Coronado E, Terrill M, Cotton RW, et al. (2012) Analytical Threshold and sensitivity: Establishing RFU threshold for Forensic DNA Analysis. J Forensic Sci 58(1): 120-129.

12. Gill P, Gusmão L, Haned H, Mayr WR, Morling N, et al. (2012) DNA commission of the International Society of Forensic Genetics: Recommendations on the evaluation of STR typing results that may include drop-out and/or drop-in using probabilistic methods. Forensic Science International: Genetics 6(6): 679-688.

13. Haned H, Slooten K, Gill P (2012) Exploratory data analysis for the interpretation of low template DNA mixtures. Forensic Sci. Int Genet 6(0): 762-774.

14. Haned H, Egeland T, Pontier D, Pène L, Gill P (2011) Estimating dropout probabilities in forensic DNA samples: a simulation approach to evaluate different models. Forensic Sci Int Genet 5: 525-531.

\section{ISSN: 2574-1241}

DOI: $10.26717 / B J S T R .2018 .12 .002213$

Emiliano Giardina. Biomed J Sci \& Tech Res

cC) (i) This work is licensed under Creative Commons Attribution 4.0 License

Submission Link: https://biomedres.us/submit-manuscript.php
15. Gill P, Puch Solis R, Curran J (2009) The low-template-DNA (stochastic) threshold--its determination relative to risk analysis for national DNA databases. Forensic Sci Int Genet 3(2): 104-111.

16. Armbruster DA, Pry T (2008) Limit of Blank, Limit of Detection and Limit of Quantitation. Clin Biochem Rev 29(Suppl 1): S49-52.

17. Kelly H Bright J, Buckleton JS, Curran JM (2014) A comparison of statistical models for the analysis of complex forensic DNA profiles. Science and Justice 54: 66-70.

18. Gill P, Brenner CH, Buckleton JS, Carracedo A, Krawczak M, et al. (2006) DNA commission of the International Society of Forensic Genetics: Recommendations on the interpretation of mixtures. Forensic Sci Int 160: 90-101.

19. Bleka Ø, Storvik G, Gill P (2016) Euro For Mix: An open source software based on a continuous model to evaluate STR DNA profiles from a mixture of contributors with artefacts. Forensic Science International: Genetics 21: 35-44.

20. Benschop CCL, van der Beek CP, Meiland HC, van Gorp AG, Westen AA, et al. (2011) Low template STR typing: effect of replicate number and consensus method on genotyping and DNA database search results. For Sci Int Genetics 5: 316-328.

21. FBI (2000) Quality assurance standards for forensic DNA testing laboratories, Forensic Science Communication 2(3).

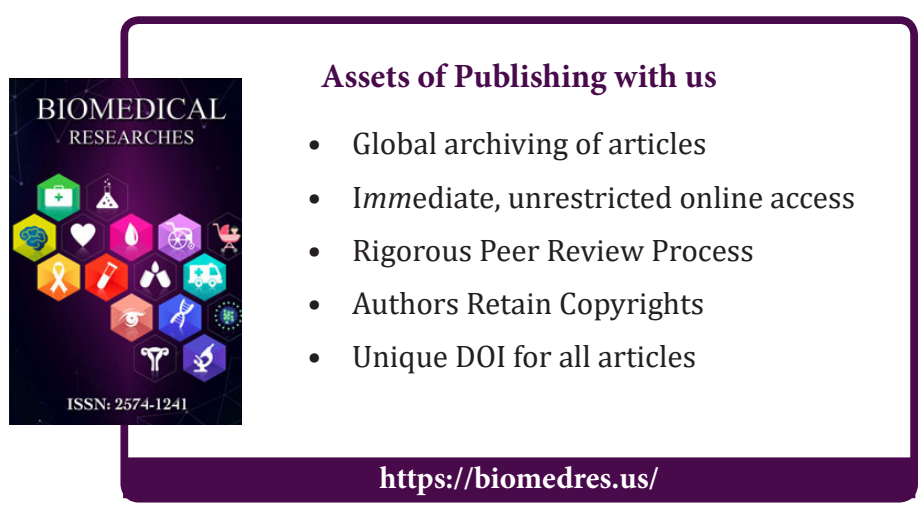

